\title{
PACS: numerical approach and evaluation of a concept for dimensioning pressure-actuated cellular structures
}

\author{
B. Gramüller ${ }^{1}$ C. Hühne ${ }^{1}$
}

Received: 18 January 2015/Revised: 7 August 2015/Accepted: 10 August 2015

(C) Deutsches Zentrum für Luft- und Raumfahrt e.V. 2015

\begin{abstract}
A biologically inspired concept is investigated which can be utilized to develop energy efficient, and lightweight adaptive structures for various applications. Summarizing basic demands and barriers regarding shapechanging structures, the basic challenges of designing morphing structures are listed. The analytical background describing the physical mechanisms of PACS is presented in detail. This work focuses on the numerical approach of calculating the geometrically, highly nonlinear deformation states of pressure-actuated cellular structures. Beyond the calculation of equilibrium states, a form-finding algorithm is presented, which allows determining structural designs following predefined target shapes. Initially made assumptions are dropped incrementally to show the effects on the accuracy of the modeling. Finite element methodbased calculations and experimental test results provide the computational target data for the varying grade of simplifications. Representative of more complex structures, like aircraft control surfaces, the examined geometries are chosen to evaluate the generic numerical methods and to validate the functionality of the basic working principle.
\end{abstract}

Keywords PACS - Shape-variable - Morphing · Form-finding $\cdot$ Cellular $\cdot$ Pressure

This paper is based on a presentation at the German Aerospace Congress, September 16-18, 2014, Augsburg, Germany.

\footnotetext{
B. Gramüller

benjamin.gramueller@dlr.de

1 DLR, Institute of Composite Structures and Adaptive Systems, Lilienthalplatz 7, Brunswick, Germany
}

\section{Introduction}

Fluidic actuators can be used to integrally combine an efficient, lightweight and accurate drive system with a deformable structure. The advantages of pneumatic and hydraulic actuators as compared to other drive systems are examined by Huber et al. [1]. The specific stresses and strains as well as the resolution of motion of these actuators lead to a wide range of use and predestinates it for aeronautical applications. In nature, the combination of fluidic actuation and a shape-variable structure can be discovered at the family of nastic plants. Representatives like the thigmonastic Cape Sundew (Drosera Capensis) and the Venus Flytrap (Dionaea Muscipula), which use their touch-sensing capabilities to trap small insects are examples for a successful implementation of biological integral morphing structures. Another common example is given by the seismonastic Mimosa Pudica that protects its fragile leafage through a folding mechanism when stimulated. Sibaoka investigated the mechanisms of nastic plants. He describes the loss and gain of turgor-internal hydrostatic cell sap pressure-(symbolized by $\mathrm{H}_{2} \mathrm{O}$ in at the upper right depiction of Fig. 1) as the driving force for the distortions [2]. Pressures of more than $8 \mathrm{MPa}$ can be reached [3].

Researchers working on form variable cellular structures made huge efforts to adapt this principle to a mechanically usable structural system. Vos et al. developed the pressure adaptive honeycomb (PAH) concept for actuating their Gurney Flap. This trailing edge flap autonomously changes its shape in different flight altitudes and takes advantage of aerostatic pressure differences [4]. By using thin-walled honeycombs, made from FAA-certifiable aluminum, a very lightweight structure results. Pagitz et al. transferred the idea of fluidic pressure driven morphing structures into a 
two-dimensional concept with a promising degree of deformation, high flexibility and sizeable characteristic [5]. Compared to the PAH concept, the main difference of PACS consists in the variable side length of its cells. Moreover, a thickened central web structure between cells of varying differential pressures is necessary for bearing pressure-induced bending moments. The utilization of foam cores in a sandwich construction could compensate the additional weight at the cell sides compared to the PAH. A PACS structure of multiple pressure dependent shapes can be mathematically deduced by manipulating the equilibrium state of each cell and thereby of the cell compound. Pagitz et al. showed with numerical methods how the deformational shape of such a structure can be controlled for multiple cells and cell rows using flexure hinges [6]. They established a form-finding approach which allows conceiving structures that vary their shapes stepless between multiple form functions.

The functional flexibility of PACS is demonstrated by the examples of a morphing airfoil and a shape adaptive backrest [7]. With their real-life implementation of a single row PACS demonstrator Gramüller et al. showed the practicability of the theoretical basis [8]. Figure 1

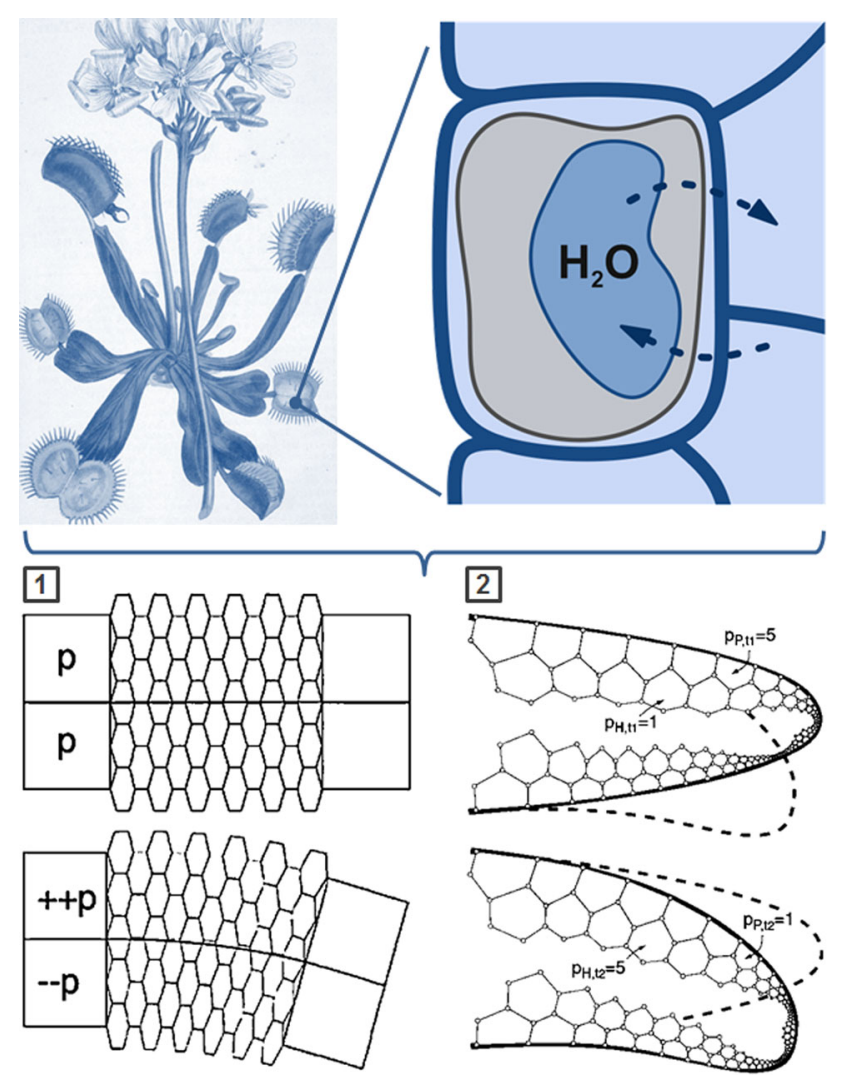

Fig. 1 Example from nature: Venus Flytrap (Dionaea Muscipula; left); Concepts of deduced operating principle: (1) PAH [14] and (2) PACS [5] summarizes the preceding work on shape-changing structures using pressurized cellular structures.

\section{Demands on adaptive structures and difficulties}

The design of conventional structures is usually driven by two groups of requirements. The first one is of programmatic manner and holds general demands like low costs, high quality and reduced development time. As a second group, structural requirements with reference to structural mechanics are determined by the expected loads and in addition by geometrical boundary conditions. These needs are also valid for shape-variable structures and a PACS structure has to withstand the design loads and simultaneously ensure to keep deformations in a tolerable range.

The actuation of shape-changing structures can be divided in two functional elements, the energy adjusting element (e.g. compressor), which transforms energy (e.g. electrical energy) from the auxiliary energy source into a usable energy form (e.g. pressure and volume) and the energy converter that modifies the received energy in order to obtain the desired energy driven effects (e.g. deformation) [9]. The special attribute about PACS is the integration of energy converter and structure as shown in Sect. 3 .

Together with the increased complexity, the overall power demands and the additional weight of the energy converter, adjusting element and peripheral sub-components like wiring, the first basic problem about shapevariable active structures appears. It can be condensed as follows: The development and implementation of a concept for shape-changing structures is only reasonable if the anticipated benefit outweighs the invested efforts. Figure 2 specifies this general demand. The energy consumption and related peripheral weight, depends on the required forces and travel ranges needed to deform the structure. Common

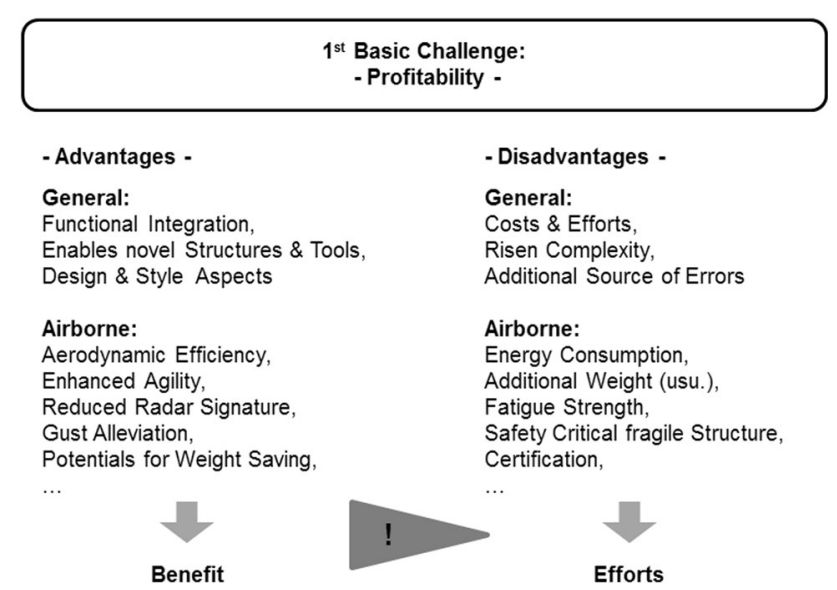

Fig. 2 Challenge of generating profitable adaptive structures 
concepts for aeronautical shape-variable structures like the horn concept [10], the ripless plain flap [11], the active flexspar actuator [12] and the vertebrate structure [13] are in need of stiff and weighty structural components to withstand aerodynamic forces. On the contrary, Barrett et al. [14] even describe the possibility of reducing structural weight by adaptive structures. An artificial muscle structure based on the pressure driven honeycomb, similarly to PACS benefits of its weight efficient structural integrated actuator and provides the non-concentrated forwarding of distributed aerodynamic loads. Structural hard points can thus be eliminated for further weight reduction and provide an additional contribution to the advantages for airborne applications.

A raise of structural stiffness increases the sufferable external forces on the corresponding structure but heightens the necessary efforts for changing the structures shape and limits the boundaries of tolerable deformation. Thus the second challenge of developing a profitable morphing concept can be formulated: an efficient concept for shapevariable structures circumvents the seeming contradiction of a specific design being stiff and flexible at the same time (see Fig. 3). There are some concepts available which have implemented this principle, like the flexible rib from Monner [15], the cellular planar morphing structure from Vasista et al. [16], the tendon-actuated compliant cellular trusses [17] or the zigzag wingbox [18]. The common principle behind these examples is a steered release of specific degrees of freedom (dofs) by integrating hinges, compliant mechanisms or linear bearings.

Other demands on the morphing structure's actuation element concern its performance-based properties, the maximum forces respectively momentums, e.g. stall torque for an electric motor, and the related travel ranges. Regarding the combination of actuator and structure, the structural response, depending on the actuators characteristics as well as on the structural stiffness and mass

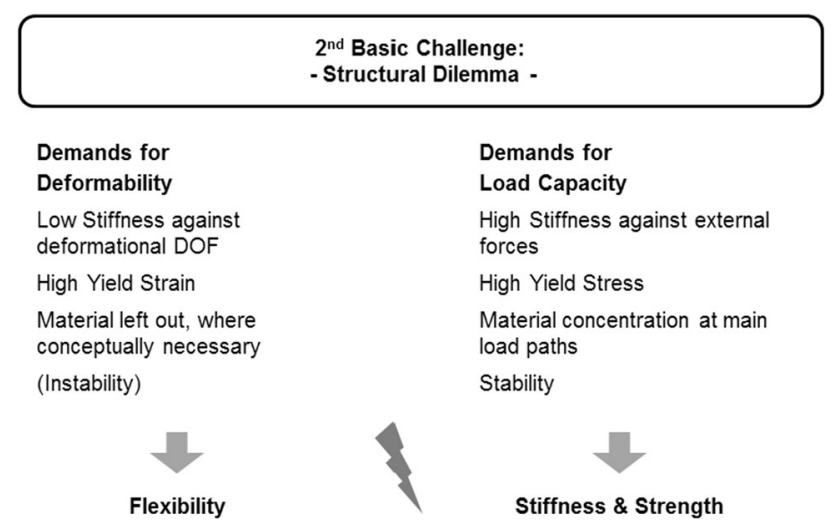

Fig. 3 Challenge of circumventing the dilemma of structural flexibility, stiffness and strength distribution, underlies the requirements for control speed and frequency and is essential for the definition of the operating range of such a concept. Other, non-unique airborne subjects as fatigue strength and certification are essential for building a real-life morphing structure. Before investigating efforts in these topics, the potentials of a concept for adaptive structures are revealed in this further step of doing research into PACS.

PACS are conceptualized to generate two-dimensional deformations on single-curved surfaces. The conceivable operating range regarding structural dimensions can be varied from centimeters to meters without having any losses of functionality, due to the possibility of adapting certain counteracting design variables. Their potential for future airborne or general structures is based on its lightweight design and energetically efficient actuation. These properties constitute a good foundation for profitable adaptive structure. The concept is further characterized by a blended structure-actuator construction, possesses a necessary minimum of stiffness in the hinge regions of the cells and generates structural stiffness through pressurization. With a high flexibility in shape variations and an adaptive structural stiffness PACS meets the second challenge of morphing concepts.

\section{Physics of PACS}

The analytical equations necessary to find and control the equilibrium state of a pressure-actuated cellular structure are essential for understanding the mechanics of this concept. Both proofing the already found numerically computed results for validity through recalculation and investigating the conceptual boundaries of a realization using compliant hinges, can be reached with the implementation of a respective algorithm. After a summary of the already published information, a continuative approach for the developed implementations is presented. The different strategy beyond that carries new aspects about handling internal and external forces as well as an alternative form-finding approach.

\subsection{Background}

The functional principle of pressure-actuated cellular structures is based on the reduction of inner energy due to volume maximization. Figure 4(1) provides a comprehensible visualization of the effects, which lead to the driving forces of this concept. Similar to a flattened balloon, a flexible membrane does not have any defined state of shape without being pressurized. Not until the balloon is loaded with a particular pressure $p_{1}$ the resulting distribution of forces lead onto bending moments and, assuming 


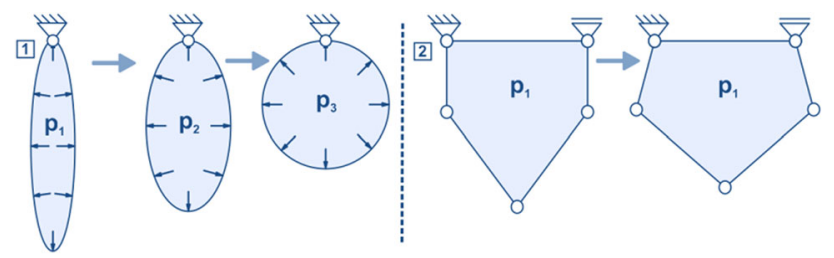

Fig. 4 Schematic diagram of (1) adiabatic expansion of pressurized fluid within flexible membrane (2) isobaric increase in volume within pressured cell

Table 1 Cell elements and associated stiffness and hinge eccentricity assumptions

\begin{tabular}{lccccc}
\hline & Variant 1 & Variant 2 & Variant 3 & Variant 4 & Variant 5 \\
\hline Cell Side & $\infty$ & $\infty$ & $\infty$ & $\mathrm{EI}$ & $\mathrm{EI}, \mathrm{EA}$ \\
Hinge & 0 & $\mathrm{EI}$ & $\mathrm{EI}$ & $\mathrm{EI}$ & $\mathrm{EI}, \mathrm{EA}$ \\
$\begin{array}{l}\text { Hinge } \\
\text { eccentricity }\end{array}$ & - & - & $\mathrm{X}$ & $\mathrm{X}$ & $\mathrm{X}$ \\
Illustration & & &
\end{tabular}

membrane characteristics, a structural shape of maximum volume. Thus the pressure is minimized $\left(p_{1}>p_{2}>p_{3}\right)$ and equally the inner energy is reduced.

As shown in Fig. 4(2) the PACS cells consist of two kinds of elements, hinges and cell sides. Depending on the level of detail used for modeling these elements, the assumptions behind the calculations lead to five major variants which are posted in Table 1. Variant one to three is part of the following chapters, variant four is exemplary and gives a prospect to the ongoing work and variant five is covered by finite element method (FEM).

In the highest level of simplification, variant 1 , the mechanical model of PACS consists of flexible hinges connecting straight cell sides of infinite stiffness. A representative cross section of such a cell is shown in Figs. 2, 3 and 4 . The inner volume of this five-edged single cell can only be enlarged by changing the angle between neighbored cell sides. The equilibrium state is again reached, when the enclosed volume is maximized. Due to the conceptual idea, the pressure stays constant during the deformation process.

Coupling an arbitrary number of cells allows superimposing the deformations of the single cells to form a shapevariable surface. Pagitz et al. showed that one cell row of pentagonal units lead to a single form function at an infinitesimal amount of pressure [5]. With the realization of a second cell row of hexagonal cells a further state of shape can be reached when only this row is pressurized.
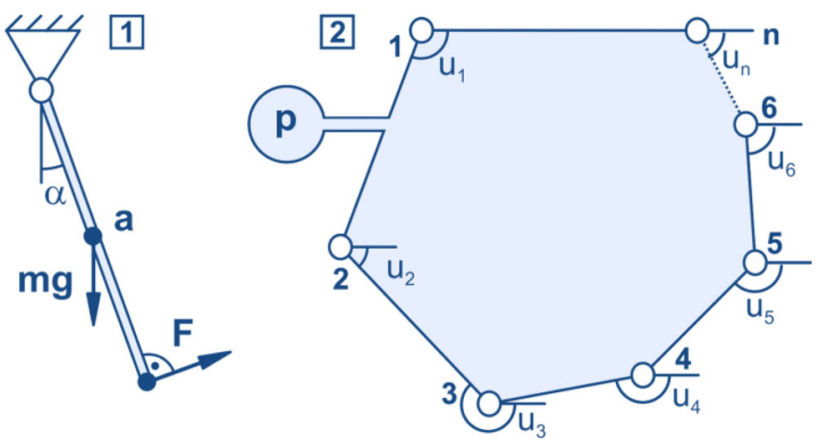

Fig. 5 Principle of virtual work, applied to a rigid cantilever and an n-edge cell

Adjusting the ratio $p_{1} / p_{2}$ between the pressure in row one $p_{1}$ and the pressure in row two $p_{2}$ at a certain value effects in a shape that ranges between these extreme form functions (see Fig. 5). A stepless transition among these states can be reached.

The mathematical approach describing PACS is used in two different ways. For a given cellular structure with cell side lengths $\boldsymbol{v}$ the equilibrium state is reached, when each infinitesimal change of the vector of hinge angles $\delta \boldsymbol{\alpha}$ causes a raise of structure inherent energy. For a given cell pressure $p$ the forces and momentums within the structure can be calculated. In order to implement a form-finding algorithm, hinge angles provide the fixed parameter for the system of equations and are determined to model the target shape. The cell side lengths are variable. Also for this approach, forces and momentums can be excerpt for the equilibrium state that depends on the respectively used pressure.

\subsection{Quotation: approach of volume maximization}

For a polygonal single cell as well as for a double row cellular structure consisting of pentagonal and hexagonal cells, the equilibrium state can be found numerically. The first approach exploits the behavior of pressurized systems to deform into a state of maximum volume $V$. This endeavor bases on the 2nd law of thermodynamics, which demand an increase of entropy $S$ for any spontaneous change of state. For a closed thermodynamic system, the amount of substance $n$ and the gas constant $R$ are invariable. At a constant temperature $T$, a decline of inner energy $\Delta U<0$ causes a raise of entropy $\Delta S$ due to the reduction of the enthalpy $\Delta H$ [19]. The Eqs. 1-3 show the relation between these values and explain how an increase of volume results in a raise of entropy.

$$
\begin{aligned}
& \Delta S=-\frac{\Delta H}{T} \\
& \Delta H=\Delta U+\underbrace{\Delta n R T}_{\text {const }}
\end{aligned}
$$


$\Delta U=-\int_{1}^{2} \underbrace{\left(p(V)-p_{u}\right)}_{\text {const }} \partial V=\left(p-p_{u}\right)\left(V_{1}-V_{2}\right)$

Pagitz et al. make use of this physical law and formulate the following equations for calculating the equilibrium state of a cellular structure. In order to present the complete numerical knowledge about PACS and since this approach is used to verify modeling variant 1 , a short summary about the approach of volume maximization is given in Eqs. (4)(11) [5].

$\boldsymbol{f}=\sum_{n=1}^{n_{P}} \boldsymbol{f}_{P n}+\sum_{n=1}^{n_{P}-1} \boldsymbol{f}_{H n}=0$

with $\boldsymbol{f}_{P n}=p_{P} \frac{\partial A_{P n}}{\partial \boldsymbol{u}_{P n}}$

and $\boldsymbol{f}_{H n}=p_{H} \frac{\partial A_{H n}}{\partial \boldsymbol{u}_{H n}}$

The global force vector $\boldsymbol{f}$ for all pentagonal cells of quantity $n_{P}$ and hexagonal cells of quantity $n_{H}$ vanishes in equilibrium. It is calculated as the sum of weighted derivatives of the cells' areas $A_{P n}$ or $A_{P n}$ with respect to the rotational degree of freedom $\boldsymbol{u}$.

$\Delta \boldsymbol{u}=-\boldsymbol{K}(\boldsymbol{u})^{-1} \boldsymbol{f}(\boldsymbol{u})$

$\boldsymbol{K}=\sum_{n=1}^{n_{P}} \boldsymbol{K}_{P n}+\sum_{n=1}^{n_{P}-1} \boldsymbol{K}_{H n}$

with $\quad \boldsymbol{K}_{P n}=p_{P} \frac{\partial^{2} A_{P n}}{\partial \boldsymbol{u}_{P n}^{2}}$

and $\quad \boldsymbol{K}_{H n}=p_{H} \frac{\partial^{2} A_{H n}}{\partial \boldsymbol{u}_{H n}^{2}}$

For the calculation of the increment $\Delta \boldsymbol{u}$ of the cell side angles at the current state, the stiffness matrix $\boldsymbol{K}$ is needed and can be found as the weighted second derivative of the respective areas. It was shown that for given cell side lengths $v$ the equilibrium state, defined by the cell side angles $\alpha$ can thus be calculated iteratively.

A separate way to reach equilibrium is to retain parts of the matrix of cell side angles and thus compute the required cell side lengths. These angles can be defined such that the structure's surface moves into a given target shape. The strategy of calculating the shape of a given structure when pressurized is thereby replaced with a form-finding algorithm [5]:

$\Delta \boldsymbol{v}=\lambda \boldsymbol{S}_{t_{1}, t_{2}}^{T} \boldsymbol{r}_{S}$

Computing the increment $\Delta v$ for the current cell side lengths $v$ and the associated cell side angles in equilibrium state allows to iteratively approaching the target shape. The factor $\lambda$ defines the step length during form finding in order to minimize the 2-norm of the residual shape vector $\boldsymbol{r}_{S}$ that comprises the difference between current and target angles. $\boldsymbol{S}_{s t_{1}, s t_{2}}^{T}$ is the sensitivity matrix coupling the change of angles with the change of cell side lengths for the two target states $s t_{1}$ and $s t_{2}$.

\subsection{Variant 1: infinitesimal hinge stiffness}

An alternative solution to the approach of volume maximization is given by the method of virtual work. As it relies on the information of hinge and cell side positions, angles and displacements, it is easy to extract element stresses and also to apply external loads. Through the more universal nature of this method, it can be modified comparatively quickly. The flexibility has however to be paid in the form of computation time. The general approach, the calculation of structural loads, the procedure of considering external forces as well as a fast converging form-finding algorithm are first illuminated on the basis of modeling variant 1 . For reduced assumptions this implementation is extended in the subsequent chapters.

\subsubsection{General approach}

A mechanical system is in equilibrium when the derivative of the potential energy $\Pi$ vanishes:

$\dot{\Pi}=-\frac{\delta W}{\delta r}=-f \stackrel{\text { equil. }}{=} 0$

The difference between the following approach and the one presented by Pagitz et al. is rooted in the calculation of potential energy. The derivative of this potential energy is equal to the introduced global force vector $f$. Implicitly using the potential energy of pressurized volumes dislocates the computational approach from the mechanical units. The theory of virtual work utilizes the forces which explicitly act on single structural elements, like in this case cell sides. It can be used for conservative forces which are present here. The application of virtual displacements $\delta r$ provides an efficient way of calculating the derivative of potential energy. A simple example shall explain the approach.

Figure 5(1) shows a flexible mounted rigid cantilever which is loaded with a beam perpendicular force $F$ and the vertical weight $\mathrm{mg}$. The virtual work $\Delta W$ is then calculated as the sum of all external forces $\boldsymbol{F}_{i}^{(e)}$ times the associated force parallel component of the virtual displacement $\Delta \boldsymbol{r}_{i}$. Equation 13 gives the solution for the depicted example and allows calculating the angle $\alpha$ for the equilibrium state (see Eq. (15)).

$\delta W=\sum_{i=1}^{n} F_{i}^{(e)} \delta \boldsymbol{r}_{i}$ 


$$
\begin{aligned}
& \delta W=\left[F-\frac{1}{2} \operatorname{mgsin}(\alpha)\right] a \delta \alpha \\
& \frac{\delta W}{\delta \alpha} \stackrel{\text { equil. }}{=} 0, \quad \text { for } \quad \alpha_{\mathrm{eq}}=\operatorname{asin}\left(\frac{2 F}{\mathrm{mg}}\right)
\end{aligned}
$$

For a pressurized single cell of $j=[m]$ cell sides the equilibrium shape can be found equally. The cell's geometry in the two-dimensional space is determined by a matrix of cell side lengths $v$ with size $[m]$ and a matrix of rotational dofs $\boldsymbol{u}$ with size $k=[m-3]$. To find the equilibrium state for a given PACS the cell side lengths provide the known and the rotational dofs or cell side angles the unknown variables. Figure 5(2) shows the notation of the variables for a single cell with pressure load $p$.

The cell side vector is

$\boldsymbol{a}_{j}=\left[\begin{array}{ll}a_{j, x} & a_{j, y}\end{array}\right], \quad$ with $\left|\boldsymbol{a}_{j}\right|=v_{j}$.

Assuming infinite stiffness for cell sides and flexible hinges the force vector is computed with the unit vector $\boldsymbol{e}_{3}$ to

$\boldsymbol{F}_{j}=p v_{j}\left[\frac{\boldsymbol{a}_{j}}{\left\|\boldsymbol{a}_{j}\right\|} \times \boldsymbol{e}_{3}\right]=p\left[\boldsymbol{a}_{j} \times \boldsymbol{e}_{3}\right]$.

The local virtual displacement vector $\delta \boldsymbol{x}$ is computed as a function of the virtual displacement at the rotational dof $\delta u_{k}$ and is illustrated in Fig. 6. It is determined as the displacement at the center of each cell side due to the virtual displacement $\delta u_{k}$ :

$\delta \boldsymbol{x}_{j, k}=g\left(\boldsymbol{v}, \boldsymbol{u}, \delta u_{k}\right)$

The function $g()$ holds the trigonometric terms, which are necessary to describe a polygon with the parameters $v$ and $\boldsymbol{u}$ that can be found in the work of Pagitz et al. [5].

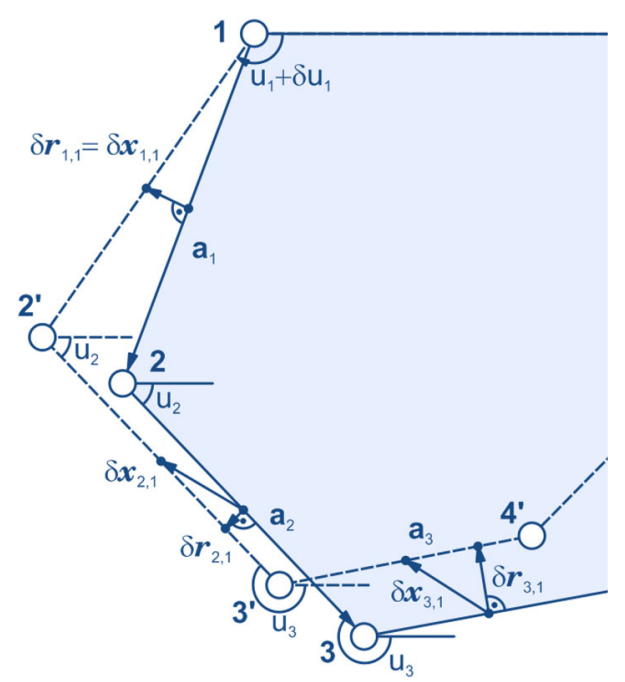

Fig. 6 Schematic description of the kinematical correlations of an m-edged single cell used for the approach of virtual work
With the additional information about the virtual displacement $\delta u_{k}$ at hinge $k$ the displacement of the point of origin for the resultant force vector $\boldsymbol{F}_{j}, \delta \boldsymbol{x}_{j, k}$ is calculated.

The vector quantity of the force parallel virtual displacement is formed by the vertical projection of the local displacement on the local force:

$\delta r_{j, k}=\frac{\delta \boldsymbol{x}_{j, k} \cdot \boldsymbol{F}_{j}}{\left\|\boldsymbol{F}_{j}\right\|^{2}}$

The first derivative of the potential energy can be computed to

$\dot{\Pi}_{k}=-\frac{\delta W_{k}}{\delta u_{k}}=-\frac{\sum_{j=1}^{m} F_{j} \delta r_{j, k}}{\delta u_{k}}=-f_{k}=0$.

In order to solve this equation and find the equilibrium shape of the cellular structure the Newton's method with quadratic convergence for this system provides a valuable approach. This iterative solution is chosen because of its flexibility with respect to an arbitrary cells and cell sides:

$x_{n+1}=x_{n}-\frac{f\left(x_{n}\right)}{\dot{f}\left(x_{n}\right)}$ (Newton's method)

The current state variable $\boldsymbol{u}_{t+1}$ for the iteration step $t+1$ results from the following equation:

$\boldsymbol{u}_{t+1}=\boldsymbol{u}_{t}-\frac{\dot{\boldsymbol{\Pi}}}{\partial \dot{\boldsymbol{\Pi}} / \partial \boldsymbol{u}}=\boldsymbol{u}_{t}-\underline{\Pi} \mathrm{K}^{-1}$

The second derivative of the potential energy is needed to calculate the stiffness matrix $\boldsymbol{K}$ (cf. Eq. 23). The size of $\boldsymbol{K}$ is $[m-3 \times m-3]$.

$$
\boldsymbol{K}=\frac{\partial \dot{\boldsymbol{\Pi}}}{\partial \boldsymbol{u}}=\left[\begin{array}{cccc}
\frac{\partial \dot{\Pi}_{1}}{\partial u_{1}} & \frac{\partial \dot{\Pi}_{1}}{\partial u_{2}} & \ldots & \frac{\partial \dot{\Pi}_{1}}{\partial u_{m-3}} \\
\frac{\partial \dot{\Pi}_{2}}{\partial u_{1}} & \frac{\partial \dot{\Pi}_{2}}{\partial u_{2}} & & \frac{\partial \dot{\Pi}_{2}}{\partial u_{m-3}} \\
\vdots & \ddots & \vdots \\
\frac{\partial \dot{\Pi}_{m-3}}{\partial u_{1}} & \frac{\partial \dot{\Pi}_{m-3}}{\partial u_{2}} & \cdots & \frac{\partial \dot{\Pi}_{m-3}}{\partial u_{m-3}}
\end{array}\right]
$$

The equilibrium state for an m-edged single cell is thus found. Applied to a cellular structure of $i=n$ cells these equations keep their validity and can be superimposed to describe more complex structures. Depending on the kind of cell combination the number of independent state variables alternates and thus the size of the stiffness matrix does. For a double row PACS structure of $i_{1}=n_{P}$ pentagonal and $i_{2}=n_{H}$ hexagonal cells, which Pagitz et al. described in their publication, the number of independent variables reduces to $\left[n_{P}\left(m_{P}-3\right)\right]+\left[n_{H}\left(m_{H}-3-2\right)+\right.$ 1] $=2 n_{P}+n_{H}+1$ what is equal to $3 n_{P}$. Figure 7 shall illuminate this assertion.

Similarly to the computation of the equilibrium for the single cell, the derivative of the potential energy is built by 


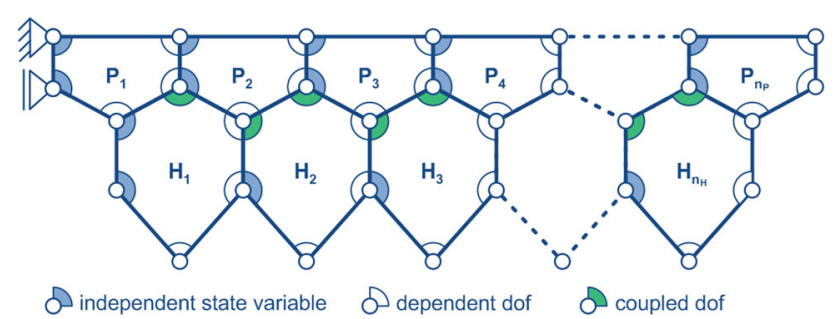

Fig. 7 Reduction of the number of independent state variables due to geometrical coupling

$\dot{\Pi}_{i, k}=-\frac{\delta W_{i, k}}{\delta u_{i, k}}=-\frac{\sum_{j=1}^{m} F_{i, j} \delta r_{i, j, k}}{\delta u_{i, k}}$.

The stiffness matrix $\boldsymbol{K}$ for this example has the size $\left[3 n_{P} \times 3 n_{P}\right]$ and can again be deduced from the derivative of the potential work after the state variable vector $\boldsymbol{u}$ :

$$
\boldsymbol{K}=\frac{\partial \dot{\boldsymbol{\Pi}}}{\partial \boldsymbol{u}}=\left[\begin{array}{cccc}
\frac{\partial \dot{\Pi}_{1,1}}{\partial u_{1}} & \frac{\partial \dot{\Pi}_{1,1}}{\partial u_{1,2}} & \ldots & \frac{\partial \dot{\Pi}_{1,1}}{\partial u_{n, m_{n}-3}} \\
\frac{\partial \dot{\Pi}_{1,2}}{\partial u_{1}} & \frac{\partial \dot{\Pi}_{1,2}}{\partial u_{2}} & & \frac{\partial \dot{\Pi}_{1,2}}{\partial u_{n, m_{n}-3}} \\
\vdots & \ddots & \vdots \\
\frac{\partial \dot{\Pi}_{n, m_{n}-3}}{\partial u_{1}} & \frac{\partial \dot{\Pi}_{n, m_{n}-3}}{\partial u_{2}} & \cdots & \frac{\partial \dot{\Pi}_{n, m_{n}-3}}{\partial u_{n, m_{n}-3}}
\end{array}\right]
$$

\subsubsection{Calculation of stresses}

For the structural design of PACS as well as for the appraisal of use-dependent practicability, stress values provide the necessary input. The computation of stresses is also processed using the method of virtual work. Equal to the virtual rotation $\delta u$ a virtual displacement $\delta v$ of cell side lengths causes the virtual work $\delta W$. The quotient of virtual work and virtual displacement yields to the force value within the observed cell side:

$\dot{\Pi}_{j}=-\frac{\delta W_{j}}{\delta v_{j}}=-\frac{\sum_{j=1}^{m} F_{j} \delta r_{j}}{\delta v_{j}}=-f_{j}=0$

Depending on the respective wall thickness $t_{j}$, the cell side stress for a PACS cell of depth $d_{j}$ is

$\sigma_{j}=\frac{f_{j}}{t_{j}}$.

For all of the subsequent depictions showing structural stresses a wall thickness of $t_{j}=1 \mathrm{~mm}$ and a depth of $d_{j}=$ $1 \mathrm{~mm}$ is underlying.

\subsubsection{External forces}

Equally to the pressure-induced forces external loads of number $n_{e}$, if present, are considered by calculating the

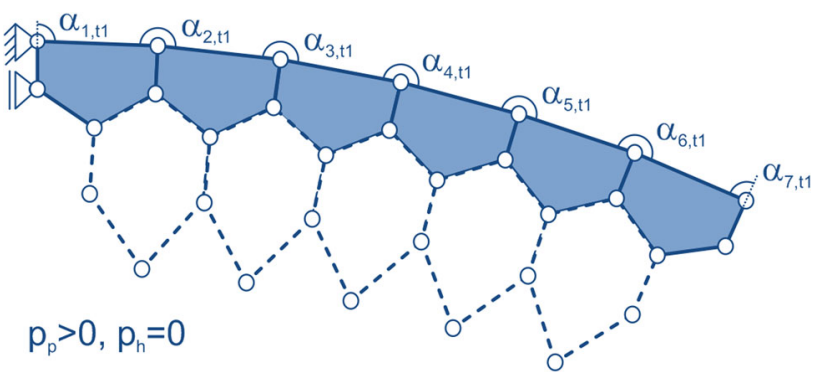

Fig. 8 Known variables at pressure set $p_{s t 1}$ for the form-finding approach

product of external force $F_{\text {ext }}$ times the related virtual displacement $\delta r_{\text {ext }}$ :

$$
\begin{aligned}
\dot{\Pi}_{k} & =-\left(\frac{\delta W_{j}}{\delta u_{k}}+\frac{\delta W_{\mathrm{ext}}}{\delta u_{k}}\right) \\
& =-\frac{\sum_{j=1}^{m} F_{j} \delta r_{j, k}+\sum_{h=1}^{n_{e}} F_{\mathrm{ext}, h} \delta r_{\mathrm{ext}, h, k}}{\delta u_{k}}=0
\end{aligned}
$$

\subsubsection{Form finding}

The difference between finding the equilibrium state of a given PACS structure and calculating the structure for a desired shape variation lies in the set of known and unknown variables. As visualized in Fig. 8 the outer shape of PACS can be defined by one angle per pentagonal cell plus one additional angle for the connector cell side of the last pentagonal cell. For a double row cantilever with two attainable shape functions at the pressure sets $s t_{1}$ and $s t_{2}$, $2\left(n_{p}+1\right)$ known variables are given. The vector of known variables is $\boldsymbol{u}_{0}$. The mixed vector of unknown state variables $\boldsymbol{w}$ whereas consists of $n_{p}-1$ pentagon and $n_{h}+1$ hexagon angles summarized in $\boldsymbol{u}_{1}$ and $4 n_{p}+1$ pentagonal and $3 n_{h}+1$ hexagonal cell side lengths $\boldsymbol{v}$.

$\boldsymbol{u}=\left[\begin{array}{l}\boldsymbol{u}_{0} \\ \boldsymbol{u}_{1}\end{array}\right] ; \quad \boldsymbol{w}=\left[\begin{array}{c}\boldsymbol{u}_{1} \\ \boldsymbol{v}\end{array}\right]$

The first derivative of the virtual work is again found according to Eq. (24) as the equilibrium state still has to fulfill Eq. (20). Thus the unknown variables add up to $9 n_{p}$ this number is three times higher than the number of equations from (20), $3 n_{p}$. The solution for a PACS which deforms into the given states of shape at a given pressure set is thus not unique. An algorithm that mathematically combines unknown variables can be used to consider manufacturing requirements or external geometrical boundary conditions.

Pagitz et al. presented a method for the form finding of PACS structures that is based on computing a sensitivity matrix which relates the change of rotational dofs to the change of cell side lengths [5]. The initial state of $\boldsymbol{u}_{0}$ is 
chosen to be identical with the manufacturing state. 2000 to 20,000 iterations are necessary to find the shape of an optimized structure with an accuracy of at least $0.01^{\circ}$ related to the target values [6].

A novel approach for solving the form-finding problem for a PACS structure reduces the necessary number of iterations significantly. In contrast to evaluating the deviation between current and target cell angles after each iteration step, the residual energy potential of the structure is used to compute the increment for the change of cell side lengths. This allows to additionally coupling the change of unknown rotational dofs $\boldsymbol{u}_{1}$ to the change of cell side lengths $\boldsymbol{v}$. For an initial state the target shapes $s t_{1}$ and $s t_{2}$ are used. The stop criterion from the approach of calculating a PACS structure's equilibrium state is still valid and leads to a maximum angular deviation towards target geometry of $1.90 \mathrm{e}-7^{\circ}$ for the example shown in Fig. 10 . The target shapes are characterized by an angular deflection of $\pm 5^{\circ}$ per pentagonal cell. The related pressure sets can be obtained from the respective depiction.

Figure 9 shows the convergence behavior in dependency of the hinge stiffness, which is introduced in the following section. The number of iterations needed to fulfill the stop criterion for the remaining virtual work is equal for calculating the equilibrium state and for form finding assuming infinitesimal hinge stiffness. As the change of the manufacturing state of the structure and thus the change of initial cell side angles are also coupled to the change of cell side lengths, a non-zero hinge stiffness does not substantially raise the necessary number of iterations.

Similar to Eq. (22) the mixed vector of unknown state variables $\boldsymbol{w}$ is computed by

$\boldsymbol{w}_{t+1}=\boldsymbol{w}_{t}-\frac{\dot{\boldsymbol{\Pi}}}{\partial \dot{\boldsymbol{\Pi}} / \partial \boldsymbol{w}}=w_{t}-\underline{\Pi} \mathcal{S}^{-1}$.

where $S$ is the sensitivity matrix which relates the change of unknown variables to the virtual work and thereby to the

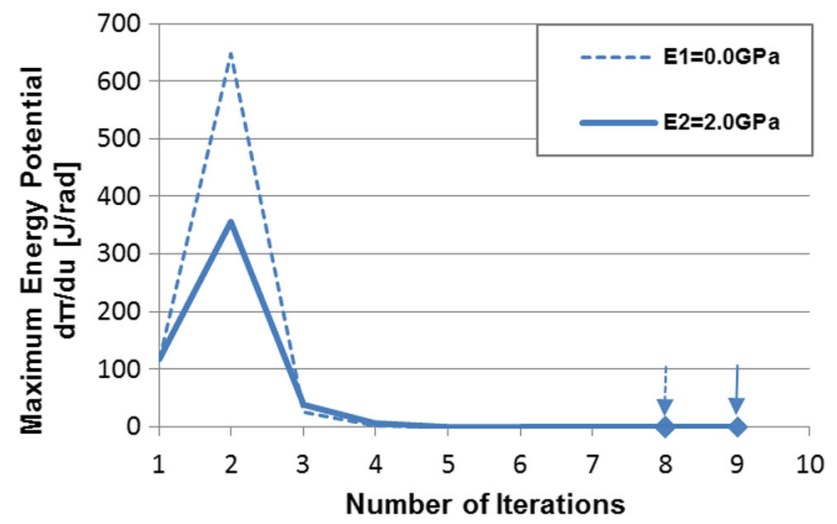

Fig. 9 Convergence curve for exemplary structure extracted from the form-finding procedure for infinitesimal and finite hinge stiffness remaining energy potential. It is calculated at the equilibrium state of $\boldsymbol{u}_{1}$, where

$\dot{\boldsymbol{\Pi}}=-\frac{\delta \boldsymbol{W}}{\delta \boldsymbol{u}_{1}}=0$,

by

$$
\begin{aligned}
\boldsymbol{S} & =\frac{\partial^{2} \boldsymbol{\Pi}}{\partial \boldsymbol{u} \partial \boldsymbol{w}}=\frac{\partial \dot{\boldsymbol{\Pi}}}{\partial \boldsymbol{w}} \\
& =\left[\begin{array}{cccccc}
\frac{\partial \dot{\Pi}_{1, s t 1}}{\partial u_{1,1}} & \ldots & \frac{\partial \dot{\Pi}_{1, s t 1}}{\partial u_{1,2 n_{p}-1}} & \frac{\partial \dot{\Pi}_{1, s t 1}}{\partial v_{1}} & & \frac{\partial \dot{\Pi}_{1, s t 1}}{\partial v_{7 n_{p}-1}} \\
\frac{\partial \dot{\Pi}_{2, s t 1}}{\partial u_{1,1}} & \cdots & \frac{\partial \dot{\Pi}_{2, s t 1}}{\partial u_{1,2 n_{p}-1}} & \frac{\partial \dot{\Pi}_{2, s t 1}}{\partial v_{1}} & & \frac{\partial}{\partial \dot{\Pi}_{2, s t 1}} \\
\vdots & \ddots & \vdots & & \ddots & \vdots \\
\frac{\partial \dot{\Pi}_{3 n_{p}-1}}{\partial u_{1,1}} & \cdots & \frac{\partial \dot{\Pi}_{3 n_{p}, s t 1}}{\partial u_{1,2 n_{p}-1}} & \frac{\partial \dot{\Pi}_{3 n_{p}, s t 1}}{\partial v_{1}} & \cdots & \frac{\partial \dot{\Pi}_{3 n_{p}, s t 1}}{\partial v_{7 n_{p}-1}} \\
\hline \frac{\partial \dot{\Pi}_{1, s t 2}}{\partial u_{1,1}} & \cdots & \frac{\partial \dot{\Pi}_{1, s t 2}}{\partial u_{1,2 n_{p}-1}} & \frac{\partial \dot{\Pi}_{1, t s 2}}{\partial v_{1}} & & \frac{\partial \dot{\Pi}_{1, s t 2}}{\partial v_{7 n_{p}-1}} \\
\frac{\partial \dot{\Pi}_{2, s t 2}}{\partial u_{1,1}} & \cdots & \frac{\partial \dot{\Pi}_{2, s t 2}}{\partial u_{1,2 n_{p}-1}} & \frac{\partial \dot{\Pi}_{2, s t 2}}{\partial v_{1}} & \cdots & \frac{\partial \dot{\Pi}_{2, s t 2}}{\partial v_{7 n_{p}-1}} \\
\vdots & \ddots & & \vdots & \ddots & \vdots \\
\frac{\partial \dot{\Pi}_{3 n_{p}, s t 2}}{\partial u_{1,1}} & \cdots & \frac{\partial \dot{\Pi}_{3 n_{p}, s t 2}}{\partial u_{1,2 n_{p}-1}} & \frac{\partial \dot{\Pi}_{3 n_{p}, t 2}}{\partial v_{1}} & \cdots & \frac{\partial \dot{\Pi}_{3 n_{p}, s t 2}}{\partial v_{7 n_{p}-1}}
\end{array}\right]
\end{aligned}
$$

The inverse of the sensitivity matrix is computed according to the Moore-Penrose method. For the present case of a non-quadratic matrix, this approach minimizes the 2-norm of $\Pi \mathcal{S}^{-1}$ and leads to stable convergence behavior.

Compared to Fig. 8 the structure depicted in Fig. 10 shows three additional elements which came up to be

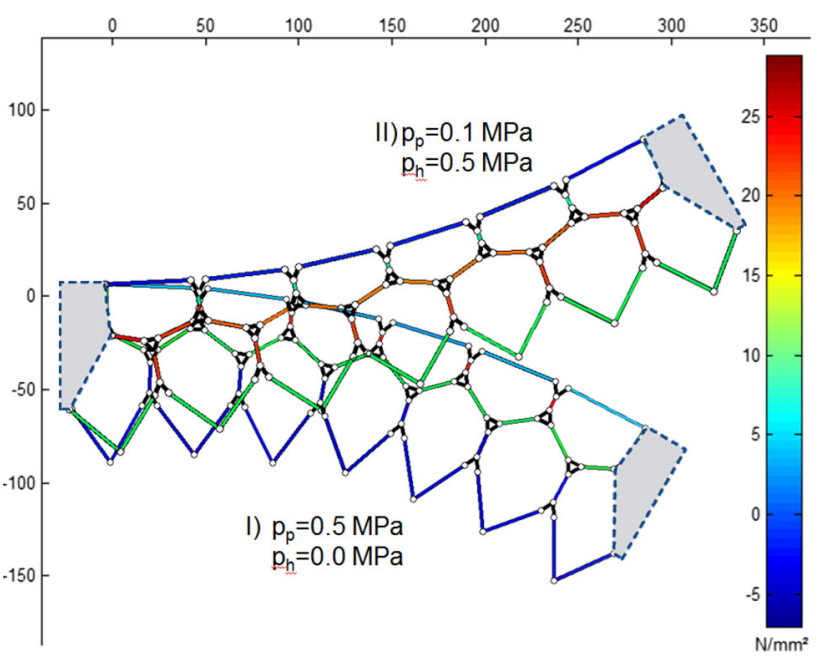

Fig. 10 Resulting structure from the form-finding procedure after nine iterations for $E=2.0 \mathrm{GPa}$ 
important during the work on the realization of a PACS structure [8]. Finite hinge stiffness and eccentric hinge positions are described in the further chapters. The connection concept at both ends of the cantilever is needed for clamping a real-life structure to its test bench or to connect multiple PACS units with each other. It is developed together with Pagitz et al. [7].

\subsection{Variant 2: finite hinge stiffness}

In contrary to the previously shown approach the cells of nastic plants do not dispose of discrete hinges of infinitesimal stiffness. Though a man-made structure can be built which most widely satisfies this assumption by using pinned hinge joints, compliant mechanisms hold two essential advantages. According to the functionality of a plant cell a compliant PACS cell is pressure-sealed in radial direction without any auxiliary structure. Beyond that, the integral design of a compliant PACS saves weight and substitutes the respective assembly process. Both, for the calculation of the pressure dependent shape of a given PACS structure and for the form-finding process, the integration of a finite hinge stiffness in the numerical model enhances the results. As this section extends the already presented approach, the equations of Sect. 3.3 are still valid and necessary.

The equivalent stiffness for a compliant hinge joint can be calculated by considering the hinge to be a beam with the flexural stiffness $E I$. This beam of length $s_{l}$ and thickness $t_{l}$ (see Fig. 11) holds the torsional stiffness $c_{l}$ at the non-coupled (independent state variables and dependent dofs, cf. Fig. 7) hinge $l$. The size of $\boldsymbol{c}$ is $\left[9 n_{p}+1\right]$. For a material of Young's modulus $E$ it results in

$c_{l}=\frac{E I_{z, l}}{s_{l}}=E \frac{t_{l}^{3}}{12 s_{l}}$.

As depicted in Fig. 11 conjugated eccentric hinges $l, 1$ and $l, 2$ are combined to locally concentrated one-dimensional hinge by:

$c_{i}=\frac{1}{1 / c_{l, 1}+1 / c_{l, 2}}$.

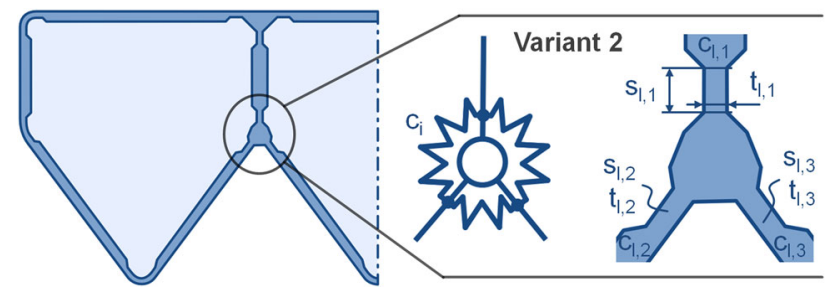

Fig. 11 Compliant hinge element with wall thickness $t$ and length $s$ and resulting bending stiffness $c$
The formula for calculating the virtual work $\delta W_{k}$ has to be extended by the approach of torsional stiffness and completed by the resulting distortion dependent momentums. The updated virtual work is

$\delta W_{k}=\sum_{j=1}^{m} F_{j} \delta r_{j, k}+\sum_{l=1}^{q} c_{l}\left(\Delta u_{t, l} \delta u_{l, k}+\frac{1}{2} \delta u_{l, k}^{2}\right)$,

with $\Delta u_{t, l}=u_{t, l}-u_{0}$.

The angular deflection $\Delta u_{t, l}$ at the iteration step $t$ is equal to the difference of the non-coupled angle $u_{t, l}$ and the manufactured hinge angle $u_{0}$ of the unloaded structure.

As $c_{l}$ depends on the structural design and is constant and the varieties of $u_{l}$ are already part of the existing calculations, the computation time is not much affected by this supplement. The approach of virtual work further allows adding this sub-formula without huge changes in the overall code.

\subsection{Variant 3: eccentric hinges}

Without a novel approach for describing mechanical element properties variant 3 provides a remedy for the assumption of locally concentrated one-dimensional hinge elements. In a real-life PACS structure the hinge length varies between five and twenty percent of the cell size. As the center of a compliant hinge not always coincides with the intersection point of linked cell sides, the dislocation of the effective hinge positions can be on the same scale. Figure 12 gives an example for unavoidable eccentricity of hinge joints. Two possibilities for the design of compliant hinges in the crossover point of three interconnected cell sides are shown for a glass fiber-reinforced plastic (GFRP) cell with a cell width of $50 \mathrm{~mm}$. It can be obtained that at

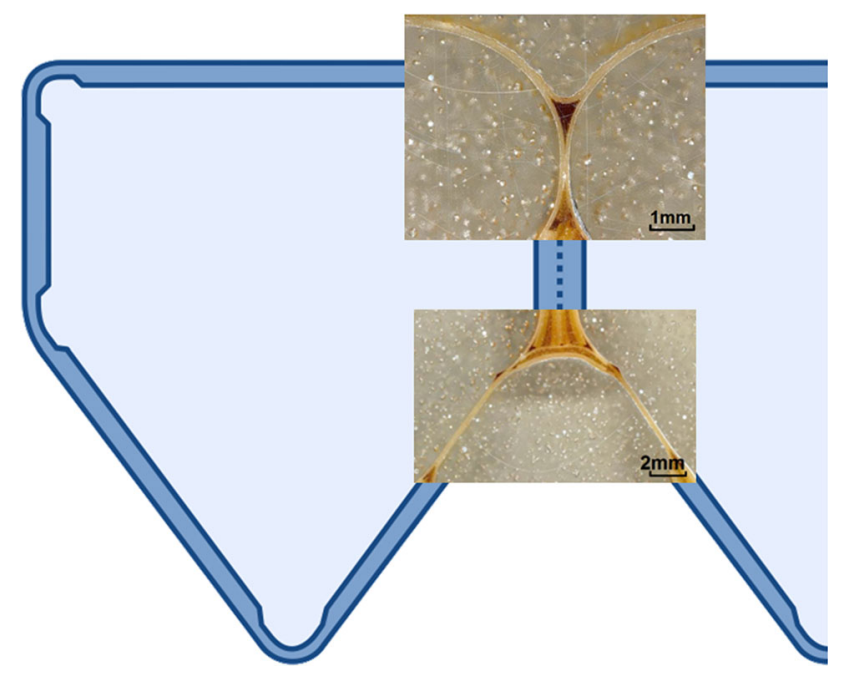

Fig. 12 Eccentric compliant hinges at crossing points of adjacent cells 


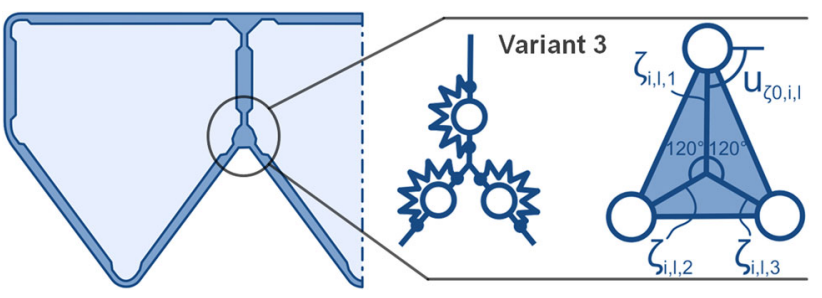

Fig. 13 Definition and notation of eccentric hinge element

this crossover an accumulation of material increases bending stiffness. The effective hinge location migrates to an eccentric position.

Overriding the approximation of concentrated hinges claims the implementation of eccentric hinge elements and leads to a more detailed and realistic model.

In order to keep the number of additional unknown variables small and considering computation efforts, the eccentric hinge is modeled as rigid triangle with fixed side lengths and only one rotational dof $\boldsymbol{u}_{\zeta}$. In the context of the approach of virtual work, a suitable way to describe this triangle is depicted in Fig. 13. The vector of eccentricity $\zeta_{i, l}$ at cell $i$ and hinge $l$ defines the geometry of the element. Together with the angle $\boldsymbol{u}_{\zeta 0}$ the initial state for the eccentric hinge is defined.

The vector $\boldsymbol{u}$ which contains the state variables for pentagonal and hexagonal cells has to be extended by rotation angle $\boldsymbol{u}_{\zeta}$. The number of independent variables thus increases by $4 n_{p}-4$ to $7 n_{p}-4$. The equations for calculating the vector of virtual work, stiffness and sensitivity matrices are still valid. The adaption of $\boldsymbol{u}$ however leads to a new size of these arrays. The form-finding approach described in Sect. 3.3.4 is also applicable for eccentric compliant hinges. Figure 10 depicts an exemplary double row PACS structure, which is calculated on the basis of variant 3 .

\subsection{Variant 5: FEM-based approach}

The benefit of the reduction of assumptions is evaluated by the comparison of the previous approaches with a more detailed modeling method. Thereby the available variants can be assessed with regard to the computation complexity. The FEM tool Ansys is used to calculate the deformations of a generic PACS structure. As this model is built of threedimensional linear solid elements including axial and bending stiffness, the FEM-based approach provides the most reliable data. The outcomes are thus used as a reference for the comparison of the alternative computational methods.

The target structure is a double row cantilever, which is designed to suit a modular concept. It consists of six pentagonal and five plus two hexagonal cells. The length of the

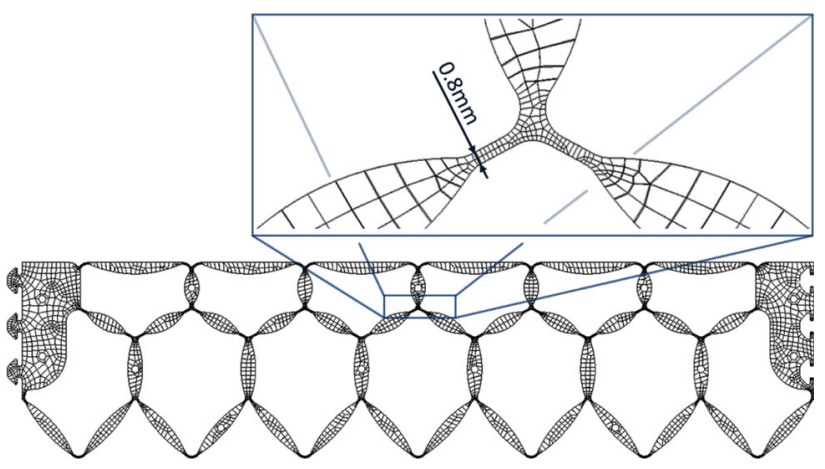

Fig. 14 Visualization of the FEM model for the modular double row cantilever

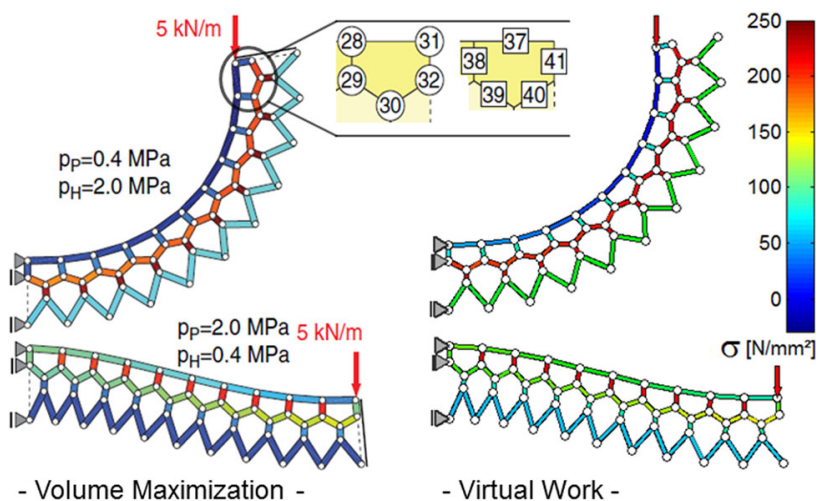

Fig. 15 Visual comparison of deformational and stress results between the approach of volume maximization-left and virtual work-right

cantilever is $350 \mathrm{~mm}$. Two separate regions are defined for meshing the structure. The cell sides elements are determined to have an element size of $2 \mathrm{~mm}$, hinge regions are modeled with a refined element size of $0.3 \mathrm{~mm}$ - see Fig. 14.

\section{Evaluation}

\subsection{Verification of variant 1}

The deformation and stress results for a loaded cantilever calculated according to variant 1 (cf. Table 1) are compared with the publicized results from Pagitz et al. [5]. Despite the completely different numerical formulations the results show good correspondence. Figure 15 pictures the deformed cantilever including cell side stresses for method of volume maximization (VM) — left and virtual work (VW) —right. Deviations of colors are due to varying imaging procedures what is made clear in following quantitative exposition. 
Table 2 Hinge coordinates at equilibrium state for volume maximization (VM) and virtual work (VW)

\begin{tabular}{llllrr}
\hline HingePos & \multicolumn{1}{l}{28} & \multicolumn{1}{l}{30} & \multicolumn{1}{l}{31} & \multicolumn{1}{l}{32} \\
\hline$x_{s t 1, \mathrm{VM}}(\mathrm{mm})$ & 634.7420 & 648.7360 & 706.0260 & 628.0109 & 677.3280 \\
$x_{s t 1, \mathrm{VW}}(\mathrm{mm})$ & 634.7526 & 648.7465 & 706.0382 & 628.0246 & 677.3419 \\
$y_{s t 1, \mathrm{VM}}(\mathrm{mm})$ & 497.7787 & 496.9998 & 553.0957 & 597.5519 & 605.7876 \\
$y_{s t 1, \mathrm{VW}}(\mathrm{mm})$ & 497.7714 & 496.9911 & 553.0862 & 597.5448 & 605.7789 \\
$x_{s t 2, \mathrm{VM}}(\mathrm{mm})$ & 884.3763 & 884.3378 & 937.0112 & 984.3722 & 989.0510 \\
$x_{s t 2, \mathrm{VW}}(\mathrm{mm})$ & 884.3754 & 884.3366 & 937.0098 & 984.3712 & 989.0498 \\
$y_{s t 2, \mathrm{VM}}(\mathrm{mm})$ & -157.5712 & -207.5713 & -236.3032 & -156.6585 & -206.4391 \\
$y_{s t 2, \mathrm{VW}}(\mathrm{mm})$ & -157.5725 & -207.5725 & -236.3046 & -156.6603 & -206.4406 \\
\hline
\end{tabular}

Table 3 Cell side stresses at equilibrium state for volume maximization (VM) and virtual work (VW)

\begin{tabular}{lrrrrr}
\hline CellSide & \multicolumn{1}{l}{37} & \multicolumn{1}{l}{38} & \multicolumn{1}{l}{40} & \multicolumn{1}{l}{41} \\
\hline$\sigma_{s t 1, \mathrm{VM}}(\mathrm{MPa})$ & -29.4297 & 33.7915 & 192.5062 & 193.2165 & 17.8448 \\
$\sigma_{s t 1, \mathrm{VW}}(\mathrm{MPa})$ & -29.4297 & 33.7915 & 192.5066 & 193.2167 & 17.8446 \\
$\sigma_{s t 2, \mathrm{VM}}(\mathrm{MPa})$ & 42.7883 & 204.0862 & 133.5500 & 131.7476 & 98.2794 \\
$\sigma_{s t 2, \mathrm{VW}}(\mathrm{MPa})$ & 42.7882 & 204.0865 & 133.5502 & 131.7478 & 98.2794 \\
\hline
\end{tabular}

For this approach of virtual work a virtual rotation of $\delta u=2 \mathrm{e}-6$ is used. $|\dot{\boldsymbol{\Pi}}|<1 \mathrm{e}-5$ is chosen as stop criterion for the iteration.

Tables 2 and 3 show the quantitative values for hinge positions and cell side normal stresses for the rightmost pentagonal cell of the depicted cantilever. The different pressurization conditions are identified by $s t_{1}$ and $s t_{2}$. The numbering of hinge points and cell sides can be obtained from Fig. 15. The maximum relative deviation of $2.18 \mathrm{e}-5$ for hinge coordinates and $1.12 \mathrm{e}-5$ for stresses results. The validity of the approach of virtual work is thus verified.

\subsection{Comparison of differing modeling variants}

Differences in accuracy of the three presented implementations utilizing the method of virtual work are illuminated in this exemplary comparison. The outcomes summarize the presented work on the numerical computation of PACS and assess the quality of the obtained results according to the concomitant efforts. The pressure set-up is chosen to cover both, a state of shape near the geometrical convergence which requires high pressures (I) and the case where the geometry is not converged and sensitive to slight pressure changes (II).

The FEM data described in Sect. 3.6 is used as reference for calculating deviations. Table 4 comprises the quantitative values for the rotational deformation at the first cell side of the sixth pentagonal cell as well as the percentage variance in relation to FEM data. An improvement of accuracy from +37.14 to $9.47 \%$ for the first pressure setting and from +85.88 to $+0.59 \%$ for the second one clearly confirms the benefit of increased modeling
Table 4 Rotational deformation at cell side one of the sixth pentagonal cell for the three presented modeling variants and deviations from FEM results

\begin{tabular}{lrrrl}
\hline & Variant 1 & Variant 2 & Variant 3 & Variant 5 \\
\hline$\Delta \beta_{6,1, p I}\left({ }^{\circ}\right)$ & 35.04 & 29.80 & 27.97 & 25.55 \\
$\eta_{V x, \mathrm{FEM}, p I}(\%)$ & +37.14 & +16.63 & +9.47 & - \\
$\Delta \beta_{6,1, p I I}\left({ }^{\circ}\right)$ & 6.32 & 2.86 & 3.42 & \multicolumn{1}{l}{3.40} \\
$\eta_{V x, \mathrm{FEM}, p I I}(\%)$ & +85.88 & -15.88 & +0.59 & - \\
\hline
\end{tabular}

complexity. Especially in low pressure regions, which are characterized by a non-converged geometrical deformation, the modeling methods including infinite hinge stiffness provide superior results. This can be explained by the stiffening of the overall structure and a decreasing sensitivity against non-pressure-induced forces with rising cell pressures. The eccentricity of the hinge points directly affects the energetic potential of the pressured cellular structure. The significant impact on the accuracy of computational results is quantified. Figure 16 visualizes the outcomes.

\subsection{Validation by experimental investigations}

The deformation results delivered by the most accurate numerical non-FEM method, the eccentric hinge approach are compared with the outcomes of the investigation of the only existing real-life PACS structure. To simplify manufacturing all of the cells are designed to have the same dimensions. A GFRP single row cantilever consisting of six cells of width $50 \mathrm{~mm}$ and length $450 \mathrm{~mm}$ results that 


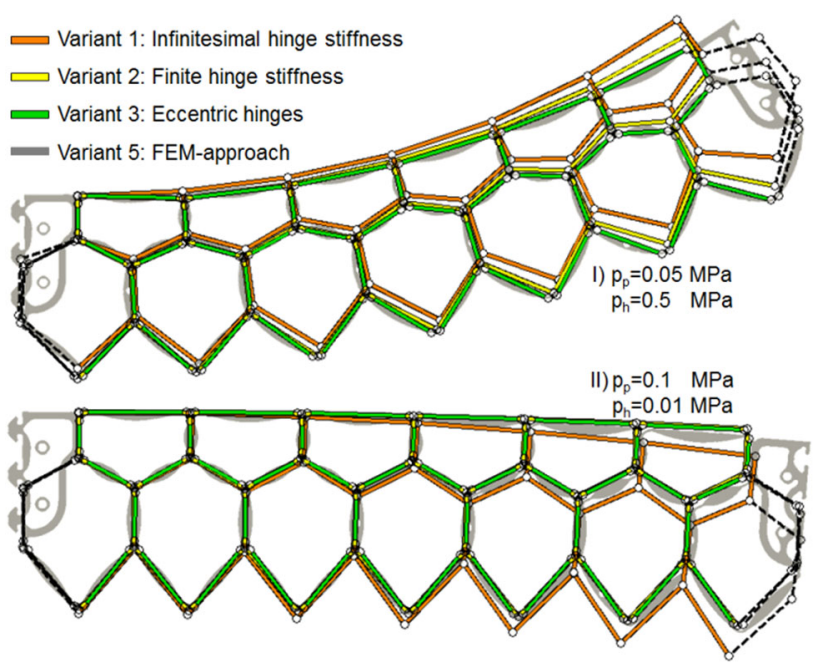

Fig. 16 Visualization of deformations from the four types of numerical computation for two different pressure set-ups

Table 5 Comparison of deformation results for the single row cantilever at cell side one of cell six at $p=0.2 \mathrm{MPa}$

\begin{tabular}{lll}
\hline$\Delta \beta_{6,1, \mathrm{ecc}}\left({ }^{\circ}\right)$ & $\Delta \beta_{6,1, \exp }\left({ }^{\circ}\right)$ & $\eta_{\text {ecc,exp }}(\%)$ \\
\hline 124.62 & 123 & +1.01 \\
\hline
\end{tabular}

reaches an entire span of $300 \mathrm{~mm}$. The main reasons for this demonstrator are to prove the theoretical methods about this concept for its practicability and to evaluate the calculation results. The authors are aware of the fact that the utilization of GFRP materials may cause problems regarding moisture intrusion due to thermal cycling. This process is intensified by the gas-tight cellular design of the PACS. As this generic investigation is not bound by the use of any specific material, also fatigue effects are not considered so far. The design process and manufacturing strategy of this prototype is part of a previous publication [8]. It shall be mentioned that the concept for pressurizing the PACS is not trivial. The solution depicted in Fig. 18 shows the tube cartridge device. It solves the related stiffness problem by utilizing an elastic silicone tube but strongly limits the applicable pressures. Further investigations on the end cap design lead to a more elegant and in particular energy efficient solution. The deformation supportive end cap (DSEC) is introduced by Gramüller et al. [20]. In favor of further examinations the physical implementation of a double row demonstrator utilizing the DSEC is presented therein.

For the given PACS geometry built from the GFRP material HexPly913 with a Young's modulus of $E=$ $42.0 \mathrm{GPa}$ an averaged hinge eccentricity of $\zeta_{i, l, x}=4 \mathrm{~mm}$, the resulting hinge stiffness of $c_{i, l}=10.938 \ldots 27.344 \frac{N}{\mathrm{rad}}$

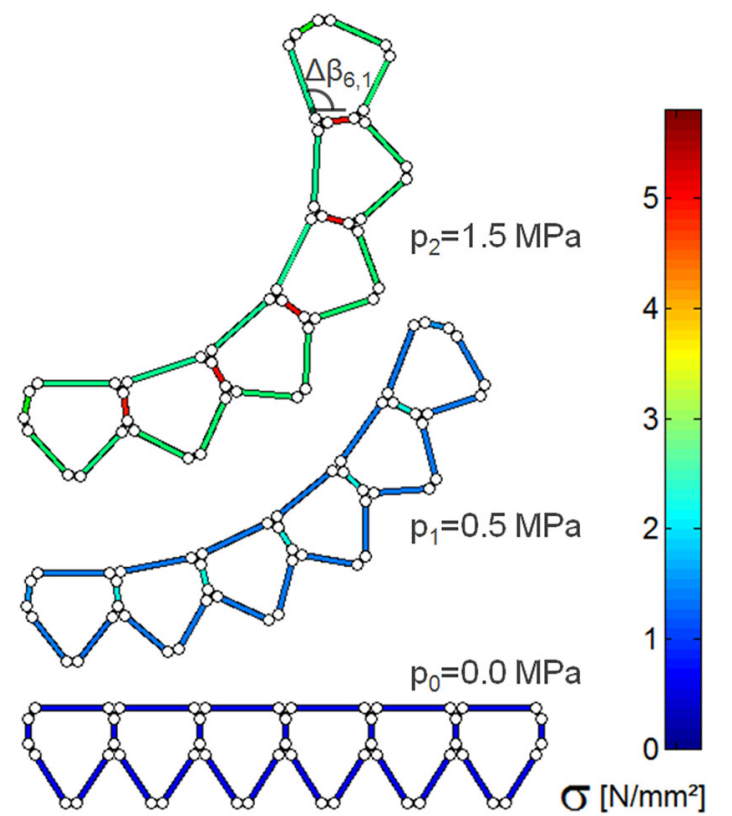

Fig. 17 Results from simulation according to the eccentric hinge approach for the single row cantilever prototype at $p_{0}=0, p_{1}=0.05$ and $p_{2}=0.15 \mathrm{MPa}$

and the pressure $p=0.2 \mathrm{MPa}$, numerical calculations are processed. Table 5 contains the deformation results for the first cell side of the sixth cell. A deviation of $1.013 \%$ shows a good match between numerical-according to variant 3 and experimental data and confirms the previous insights. The experimental value is measured with an analogue protractor.

The deformation results as well as normalized cell side stresses for the cell side thickness of $1 \mathrm{~mm}$ are depicted in Fig. 17. The related photographs of the prototype demonstrator can be compared in Fig. 18.

\section{Discussion of results}

The method of virtual work provides an alternative solution to the approach of volume maximization. Solving the geometrical highly nonlinear problem of a pressurized PACS structure can be used for both the computation of equilibrium shape and for form finding. Three different approaches with an increasing level of model accuracy are presented and their results are compared with FEM-based outcomes and experimentally achieved values. Two substantial aspects about these results shall be discussed.

As it can be obtained from Fig. 12 in a kinematical structure where compliant mechanisms are used to realize hinge joints, the determination of the position of effective pivot points is not trivial. Depending on the hinge's geometry and loading, this location shifts relatively to the 

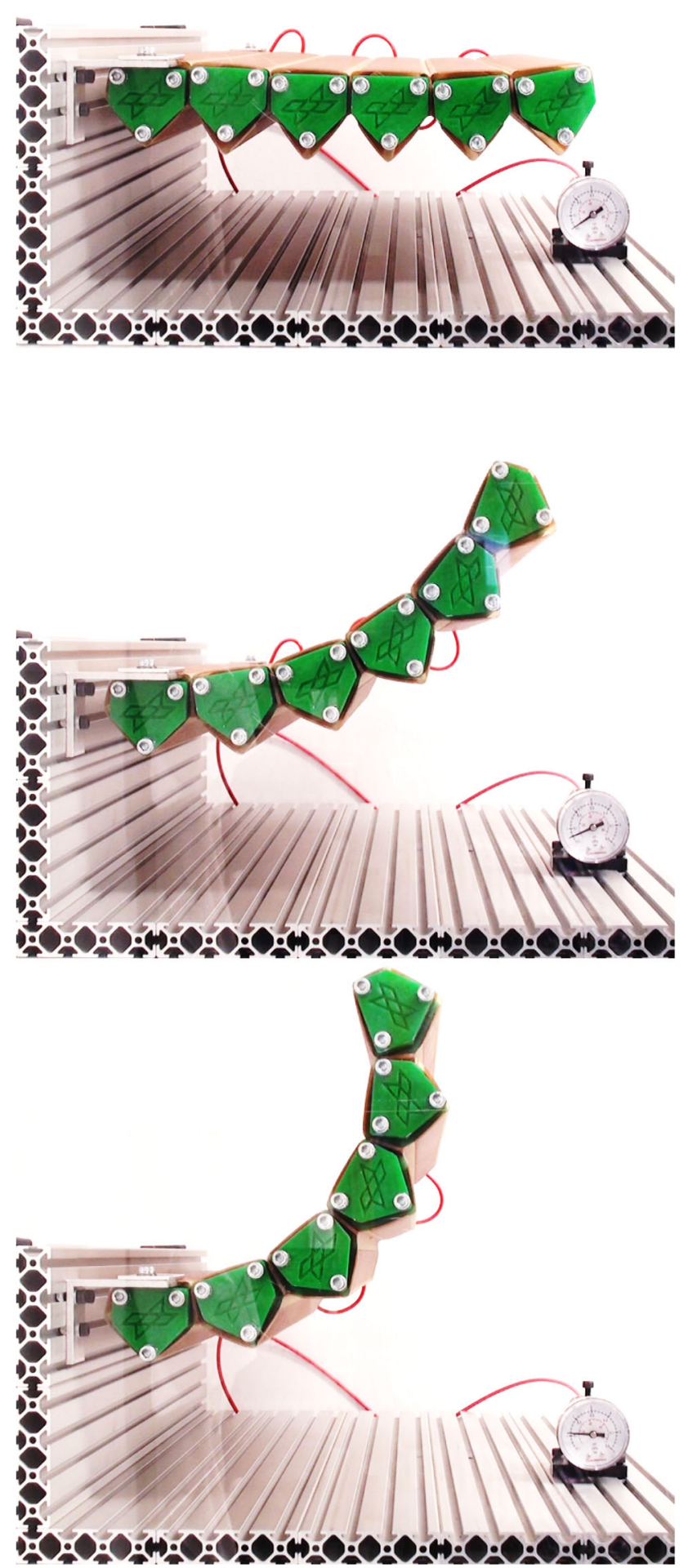

Fig. 18 Demonstrator "Single Row Cantilever" at $p_{0}=0, p_{1}=0.05$ and $p_{2}=0.15 \mathrm{MPa}$ [8]

adjacent cell sides. Concerning the more detailed computational methods with increased accuracy, the extraction of the hinge stiffness and eccentricity of a given structure is not trivial. Within a real PACS structure the gradual transition between hinge and cell side elements complicates the definition of the hinge stiffness according to Eq. (33) and the related eccentricity $\zeta$. Simulating the load dependent deformation behavior of each compliant hinge joint may provide relief and give additional insight in this relationship.

With the implementation of variant 3 , the consideration of eccentric hinges, some assumptions could be dropt but others are still implemented. Beyond the theme of concentrated hinges, the axial and bending stiffness of cell sides as well as the axial stiffness of hinge elements is not considered. Further numerical approaches may profit from the implementation of these open issues. Though the presented methods yet show good accordance with FEMbased computations and experimental investigations.

\section{Conclusion}

The most important demand on each shape-variable structure is defined by the imperative need for improvement. Therefore, the demands on the concept of pressureactuated cellular structures are investigated. The existing numerical theory about PACS is summarized and confirmed by a novel approach using the method of virtual work. Two advanced variants were presented extensively which increase the level of detail within the numerical model by first dropping the assumptions of infinitesimal hinge stiffness and subsequently of centric hinges. In comparison to a FEM calculation the different modeling variants achieved varying degrees of accordance for the two calculated states of internal pressure. With a deviation of 9.47 and $0.59 \%$ in angular deflection for different pressures, the numerical approach using eccentric hinges provides the most accurate results. Thereby it is approved that the increased modeling and computational effort enhances the quality of the results.

A single row PACS prototype consisting of six equally shaped pentagonal cells is used to demonstrate the functionality of the concept and to validate the computed data. The compliance regarding the accuracy of deformational results between eccentric hinge model and experimentally measured values lies at about $1 \%$ for this investigation.

The discussion of results shows that additional investigations on the compliant hinge elements, which allow deriving accurate descriptive parameters would improve the numerical model. An increase in the level of detail by dropping further modeling assumptions would also have a positive effect. This can be reached by the consideration of axial stiffness for hinge and cell side elements as well as by the consideration of the bending stiffness of cell sides. 


\section{References}

1. Huber, J.E. et al.: The selection of mechanical actuators based on performance indices. Proc. R. Soc. A Math. Phys. Eng. Sci. 453(1965), 2185-2205 (1997)

2. Sibaoka, T.: Rapid plant movements triggered by action potentials. Bot. Maga Tokyo 104(1), 73-95 (1991)

3. Howard, R.J. et al.: Penetration of hard substrates by a fungus employing enormous turgor pressures. Proc. Natl. Acad. Sci. U.S. 88(24), 11281-11284 (1991)

4. Vos, R. et al.: Pressure adaptive honeycomb: mechanics, modeling, and experimental investigation. In: 51st AIAA/ASME/ ASCE/AHS/ASC Structures, Structural Dynamics and Materials Conference. Orlando. Art.Nr.: 2010-2664 (2010)

5. Pagitz, M. et al.: Pressure-actuated cellular structures. Bioinspiration Biomim. 7(1), 016007 (2012)

6. Pagitz, M. et al.: Compliant pressure actuated cellular structures (2014). Online cited 2015 January 01. Available from: http:// arxiv.org/abs/1403.2197v1

7. Pagitz, M. et al.: A modular approach to adaptive structures. Bioinspiration Biomim. 9(4), 046005 (2014)

8. Gramüller, B. et al.: PACS-realization of an adaptive concept using pressure actuated cellular structures. Smart Mater. Struct. 23(11), 115006 (2014)

9. Janocha, H. (ed.): Aktoren-Grundlagen und Anwendungen, 1st edn. Springer, Berlin (1992)

10. Mueller, D.: Das Hornkonzept-Realisierung eines formvariablen Tragflügelprofils, 1st edn. Frankfurt (2000)

11. Bauer, C.: Die Rippenlose Wölbklappe-Realisierung eines formvariablen Tragflügelprofils zur aerodynamischen
Leistungsoptimierung zukünftiger Verkehrsflugzeuge, 1st ed. Bauer, C. (ed.). Shaker Verlag, Aachen (2000)

12. Barrett, R. et al.: Missile flight control using active flexspar actuators. Smart Mater. Struct. 5(2), 121-128 (1996)

13. Elzey, D. et al.: A bio-inspired, high-authority actuator for shape morphing structures. In: Proceedings Of SPIE SPIE-The International Society for Optical Engineering: Smart Structures and Materials 2003 Active Materials: Behavior and Mechanics, pp. 92-100. San Diego (2003)

14. Barrett, R. et al.: Biomimetic FAA-certifiable, artificial muscle structures for commercial aircraft wings. Smart Mater. Struct. 23(7), 074011 (2014)

15. Monner, H.P.: Realization of an optimized wing camber by using formvariable flap structures. Aerosp. Sci. Technol. 5(7), 445-455 (2001)

16. Vasista, S. et al.: Topology-optimized design and testing of a pressure-driven morphing-aerofoil trailing-edge structure. AIAA J. 51(8), 1898-1907 (2013)

17. Ramrakhyani, D. et al.: Aircraft structural morphing using tendon-actuated compliant cellular trusses. J. Aircr. 42(6), 1615-1621 (2005)

18. Ajaj, R.M. et al.: The Zigzag wingbox for a span morphing wing. Aerosp Sci Technol. 28(1), 364-375 (2013)

19. Mortimer, E. et al.: Chemie: Das Basiswissen der Chemie, 12th edn. Thieme, Stuttgart (2007)

20. Gramüller, B. et al.: Shape-variable seals for pressure actuated cellular structures. Smart Mater. Struct. 24(9), 095005 (2015) 